\title{
Brown Tumor With Spine Involvement at Multiple Levels in a Hemodialysis Patient
}

\author{
Victoria Ghernautan ${ }^{1}$, Zarwa Idrees ${ }^{1}$, Mahmoud Nassar ${ }^{1}$, Camelia Ciobanu ${ }^{2}$, Adesh Ramdass ${ }^{3}$ \\ 1. Internal Medicine, Icahn School of Medicine at Mount Sinai/NYC Health+Hospitals Queens, New York, USA 2. \\ Medicine, St. Barnabas Hospital, The Bronx, USA 3. Medicine, Icahn School of Medicine at Mount Sinai/NYC \\ Health+Hospitals Queens, New York, USA
}

Corresponding author: Victoria Ghernautan, vicutag@yahoo.com

\begin{abstract}
Brown tumor of the bone or osteitis fibrosa cystica is a rare manifestation of hyperparathyroidism, most seen nowadays in association with secondary and tertiary hyperparathyroidism. Chronic kidney disease (CKD) and end-stage renal disease (ESRD) are the major culprits of secondary hyperparathyroidism (sHPTH). CKD is known to cause phosphate retention and a decrease in 1,25-dihydroxyvitamin D and ionized calcium levels, which in turn trigger the PTH secretion. Brown tumor can affect the jawbones, femur, sternum, ribs, and rarely the spine. We present the case of a 60-year-old male with ESRD on hemodialysis who was found to have lytic bone lesions in the thoracic and lumbar spine. Initially, malignancy was suspected. Blood work revealed markedly elevated PTH at 3,563 pg/mL, hypocalcemia, and hyperphosphatemia. Biopsy of the L5-S1 lesion was consistent with reactive changes due to SHPTH. Once a diagnosis of the brown tumor was confirmed, the patient was started on cinacalcet and was referred for parathyroidectomy.
\end{abstract}

Review began 07/22/2021 Review ended 08/02/2021 Published 08/08/2021

\section{(c) Copyright 2021}

Ghernautan et al. This is an open access article distributed under the terms of the Creative Commons Attribution License CC-BY 4.0, which permits unrestricted use, distribution, and reproduction in any medium, provided the original author and source are credited.
Categories: Endocrinology/Diabetes/Metabolism, Internal Medicine, Nephrology

Keywords: brown tumor, secondary hyperparathyroidism, bone lytic lesions, chronic kidney disease, parathyroid hormone, osteitis fibrosa cystica, vertebral brown tumor

\section{Introduction}

Metabolic bone disease in chronic kidney disease (CKD) is a result of several abnormalities in calcium, phosphorus, and vitamin D metabolism. Decreased ionized calcium and hyperphosphatemia stimulate parathyroid hormone (PTH) secretion, promoting the development of secondary hyperparathyroidism (sHPTH) [1]. Persistently elevated PTH increases osteoclastic activity, bone resorption, and breakdown resulting in osteitis fibrosa cystica aka brown tumor [2]. Brown tumor is a rare complication of CKD, especially in the Western world, due to the early detection and treatment of hyperparathyroidism. It can occur in any bone; however, spine involvement is exceedingly rare [3-5]. Here, we describe a rare case of brown tumor involving the thoracic and lumbosacral spine in a patient on hemodialysis for end-stage renal disease (ESRD).

\section{Case Presentation}

A 60-year-old African American male with a past medical history of essential hypertension, hyperlipidemia, coronary artery disease, bilateral knee osteoarthritis, ESRD on hemodialysis three times a week and uncontrolled sHPTH presented to the emergency room complaining of back pain for a week after a fall. The pain was sharp, constant, radiating to the bilateral hips. The pain was worse with movement and was alleviated by acetaminophen. The patient reported compliance with the scheduled hemodialysis treatments. His home medication regimen consisted of low-dose aspirin, calcitriol $0.25 \mathrm{mcg}$ daily, furosemide $40 \mathrm{mg}$ twice a day, hydralazine $100 \mathrm{mg}$ every eight hours, nifedipine ER $90 \mathrm{mg}$ daily, and sevelamer $800 \mathrm{mg}$ three times a day.

There were lytic bone lesions in the seventh rib, T9, T11, and L1 vertebrae incidentally found on a CT chest two months prior during an ER visit. The patient was referred to an oncology outpatient clinic. He did not make it to the oncology clinic as he needed medical attention for the back pain.

On examination, midline lower back tenderness was elicited, and no neurological deficits were found. The presence of the lytic lesions on previous CT prompted our team to suspect metastatic cancer or multiple myeloma. Oncology service was consulted and recommended an urgent MRI of the lumbar spine due to the concern for cord compression, and CT of the abdomen and chest with contrast to localize other potential bone lytic lesions. They also recommended a biopsy of the most accessible lytic lesion.

Blood work showed normocytic anemia, normal leukocytes, elevated blood urea nitrogen (BUN) and creatinine, mildly elevated kappa and lambda free light chains, normal serum protein electrophoresis, normal prostate-specific antigen (PSA), normal liver enzymes, and bilirubin. Additional tests showed decreased ionized calcium (1.12 mmol/L), markedly elevated PTH at 3,563 pg/mL, alkaline phosphatase 183 


\section{Cureus}

$\mathrm{U} / \mathrm{L}(\mathrm{N}, 40-129 \mathrm{U} / \mathrm{L})$, and phosphorus $6.9 \mathrm{mg} / \mathrm{dL}(\mathrm{N}, 2.5-4.5 \mathrm{mg} / \mathrm{dL})$.

MRI of the lumbar spine showed no evidence of cord compression. Abdominal and chest CT identified the lytic lesions in the thoracic T9, T11 (Figures 1, 2), lumbar L1, L5 (Figure 3), and sacral S1 spine, and the seventh rib, which were described as suspected brown tumors but no other findings to support primary cancer or organ metastases. Total body bone scintigraphy was strongly suggestive of metabolic bone disease.

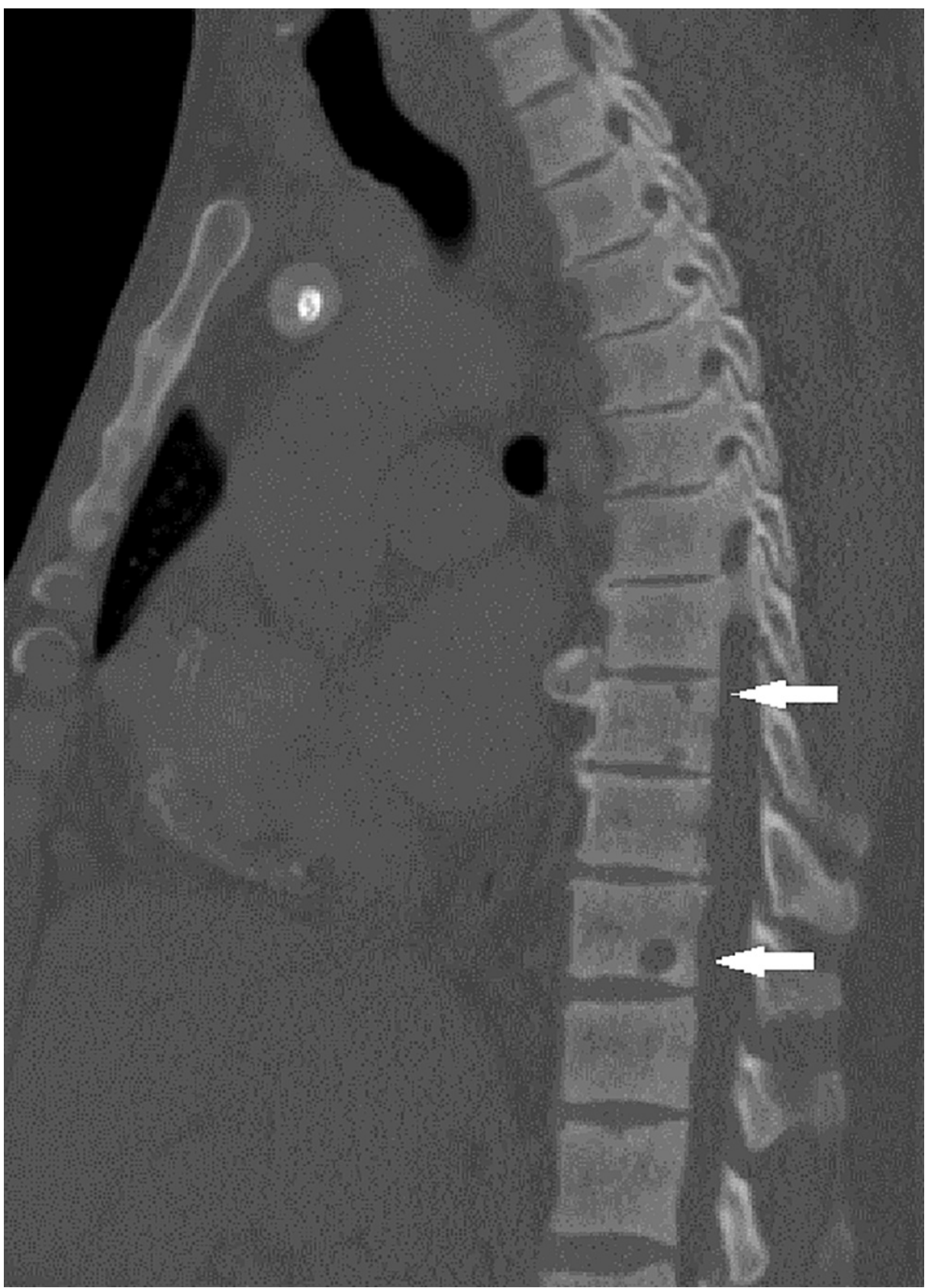

FIGURE 1: CT of the chest with contrast showing the bone lytic lesions in the thoracic vertebrae. 


\section{Cureus}

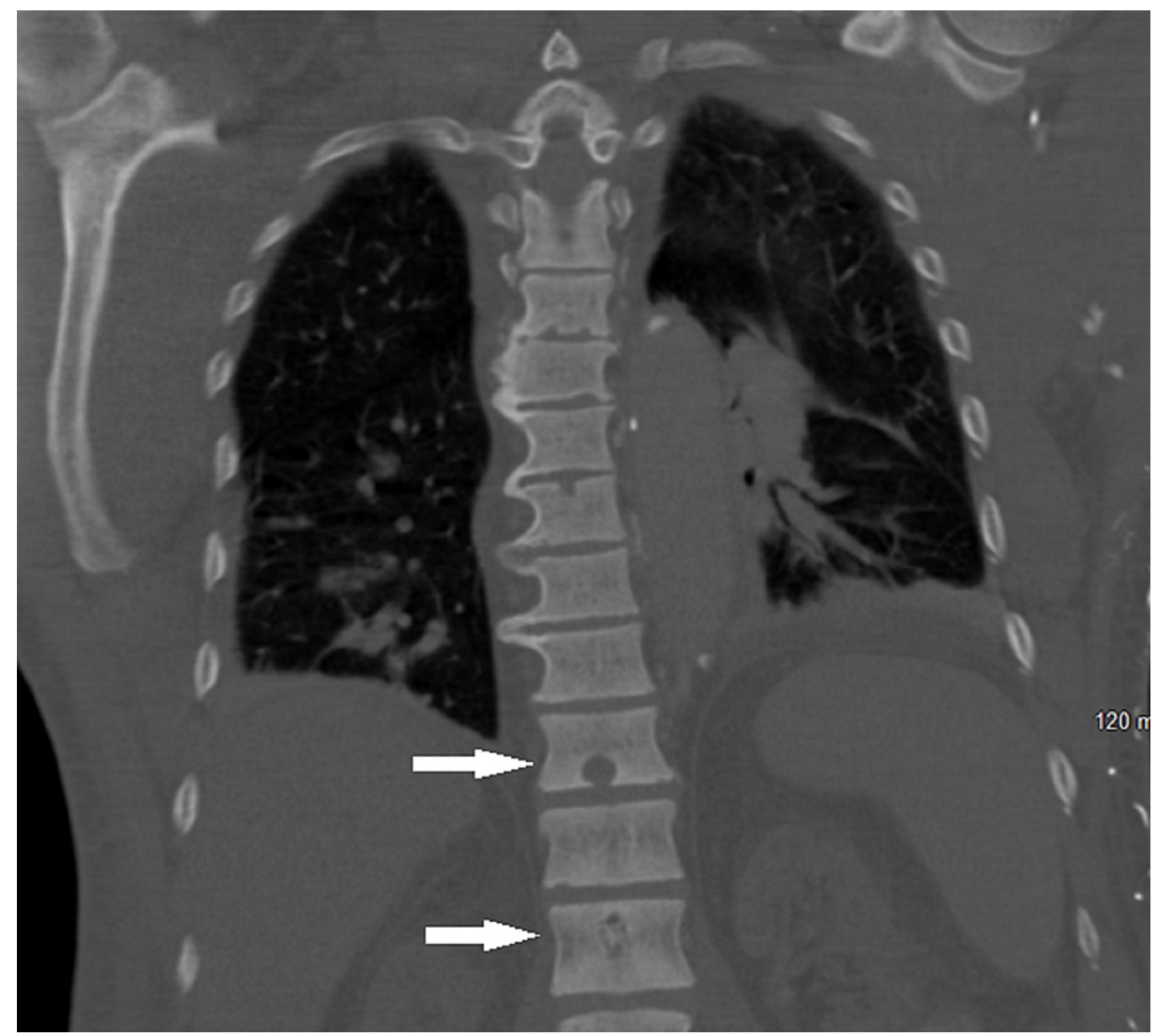

FIGURE 2: CT of the chest with contrast showing the bone lytic lesions in the thoracic vertebrae and L1 vertebra. 


\section{Cureus}

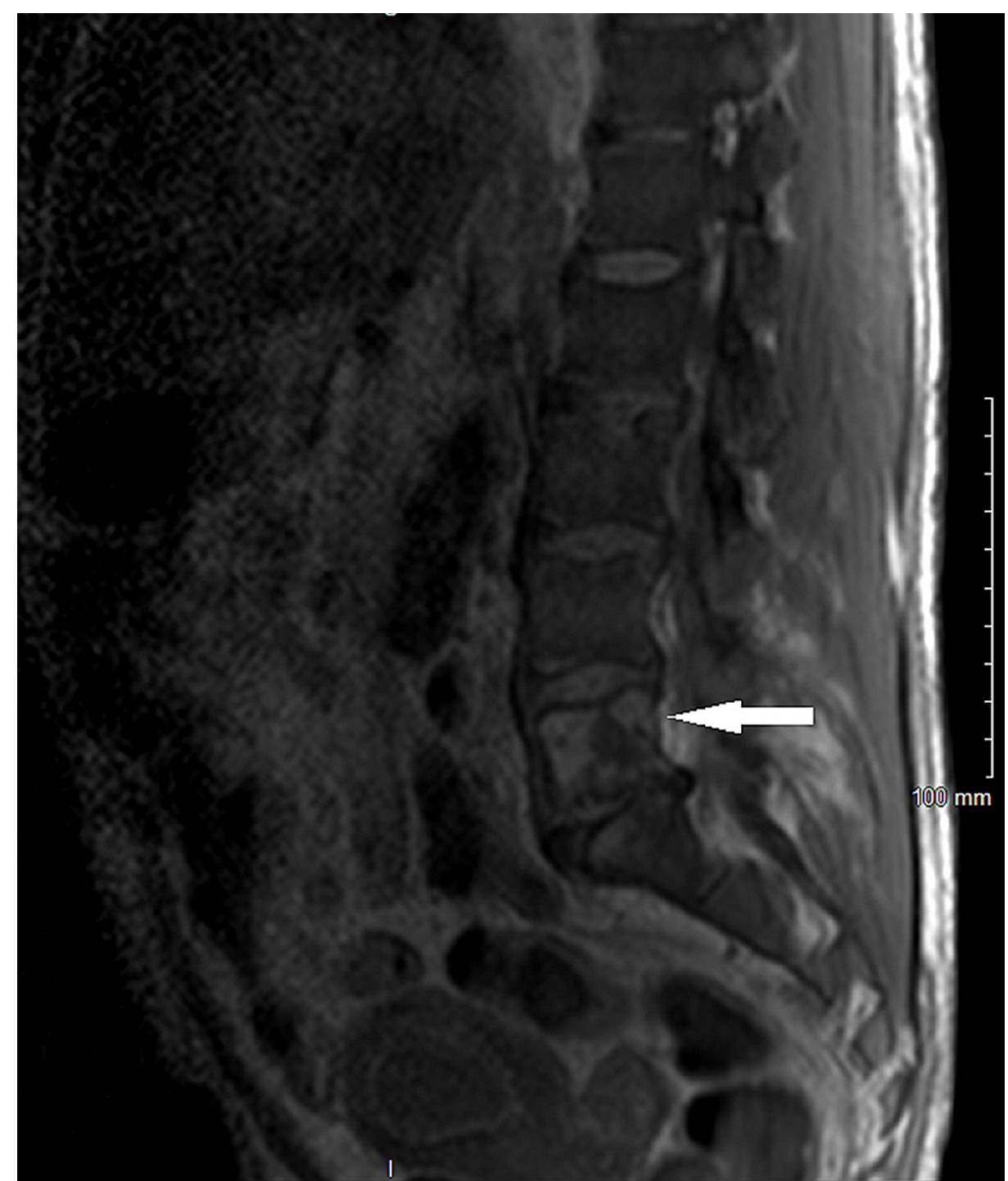

\section{FIGURE 3: MRI of the lumbar spine without contrast showing the L5}

lytic lesion.

Ultrasound-guided fine-needle aspiration of the L5-S1 lytic lesion showed chronic inflammation, fibrosis, and spindle cells consistent with reactive changes. The clinical picture along with the radiographic findings and cytology was supportive of brown tumor diagnosis and less likely of malignancy such as multiple myeloma or giant-cell tumor of the bone.

Nephrology service was also involved in patient's care and they recommended to continue the phosphate binder and switch to cinacalcet from calcitriol. He was also referred for parathyroidectomy.

The patient followed up in the primary care clinic, he was still complaining of back pain. He has a scheduled appointment with ENT for evaluation for parathyroidectomy.

\section{Discussion}

ESRD is one of the most common causes of sHPTH and can lead to the so-called "renal osteodystrophy." It includes several manifestations such as osteitis fibrosa cystica, osteomalacia, osteosclerosis $[4,6]$. Osteitis fibrosa cystica can present as lytic or sclerotic bone lesions secondary to severe bone resorption due to persistently high levels of PTH [4]. They are called brown tumors and occur more often in the jaw, ribs, sternum, and long bones. Several cases of spine involvement were described; however, they are rare [3-5]. Moreover, multilevel vertebral brown tumors are extremely rare [7]. In certain cases, they can be recurrent [8]. The first case of a brown tumor of the spine secondary to primary hyperparathyroidism (pHPTH) was published in 1968 [6].

Brown tumor is a benign and reactive lesion secondary to disrupted bone remodeling, which can occur in 
both pHPTH and sHPTH. It develops in patients with extremely elevated PTH levels for a prolonged period of time $[2,9]$. The incidence of brown tumors in ESRD patients on HD is up to $13 \%[4,9,10]$, as opposed to $1.5 \%$ in $\mathrm{PHPTH}[9]$.

Brown tumors exhibit slow growth, and their clinical presentation depends on anatomical location [3]. Our patient had several vertebrae affected in the thoracic and lumbosacral spine of unknown duration, and his symptoms of back pain started after a fall. We suspect his clinical course was indolent, and the recent fall prompted radiographic examination. CT and MRI of the spine revealed multiple radiolucencies, which were incidental findings.

The lytic bone lesions caused by brown tumors can easily be confused for an underlying neoplastic disease. Indeed, it can imitate multiple myeloma, giant cell granuloma, or metastases $[2,10]$. However, brown tumors do not exhibit malignant potentiality [10]. Therefore, malignancy was high on our differential diagnosis list, and ruling it in or out was a priority. The final diagnosis is made by tissue examination [4], which commonly reveals increased osteoclastic activity, multinucleated giant cells, fibroblast proliferation, and hemosiderin deposition $[3,4]$. The loose connective tissue is replaced by cortical and trabecular bone [6].

Medical management of the brown tumor consists of treating the SHPTH with phosphate binders, vitamin D analogs, and calcimimetics, and limiting dietary phosphorus intake [6]. If medical treatment fails, then the patient should be referred for parathyroidectomy [6]. Bisphosphonate use was reported in some cases of spinal implication before the surgical intervention to potentially suppress an increase in the tumor size post-parathyroidectomy. The ultimate treatment is subtotal or total parathyroidectomy, which prompts remineralization of the bone in months to a few years [4].

Without appropriate treatment, the brown tumor can cause bone destruction, fractures [10], and if localized in the spine, cord compression can occur with severe neurological deficits requiring surgical decompression $[4,9]$.

\section{Conclusions}

Diagnosis of brown tumors can be easily overlooked since their appearance can mimic more serious diseases, such as malignancy. A biopsy is a useful procedure that helps distinguish a benign lesion from a neoplasm and can guide the appropriate treatment. Our patient had multilevel spine involvement, which in some instances can lead to spinal cord compression. Timely detection and treatment are essential.

\section{Additional Information \\ Disclosures}

Human subjects: Consent was obtained or waived by all participants in this study. Conflicts of interest: In compliance with the ICMJE uniform disclosure form, all authors declare the following: Payment/services info: All authors have declared that no financial support was received from any organization for the submitted work. Financial relationships: All authors have declared that they have no financial relationships at present or within the previous three years with any organizations that might have an interest in the submitted work. Other relationships: All authors have declared that there are no other relationships or activities that could appear to have influenced the submitted work.

\section{References}

1. Tomasello S: Secondary hyperparathyroidism and chronic kidney disease . Diabetes Spectrum. 2008, 21:1925. 10.2337/diaspect.21.1.19

2. Khan A, Bilezikian J: Primary hyperparathyroidism: pathophysiology and impact on bone. CMAJ. 2000, 2:184-7.

3. Altan L, Kurtoğlu Z, Yalçinkaya U, Aydinli U, Ertürk E: Brown tumor of the sacral spine in a patient with low-back pain. Rheumatol Int. 2007, 28:77-81. 10.1007/s00296-007-0380-z

4. Mirzashahi B, Ardakani MV, Farzan A: Brown tumor of lumbar spine in chronic renal failure: a case report . Acta Med Iran. 2014, 52:484-7.

5. Mateo L, Massuet A, Solà M, Andrés RP, Musulen E, Torres MCS: Brown tumor of the cervical spine: a case report and review of the literature. Clin Rheumatol. 2011, 30:419-24. 10.1007/s10067-010-1608-y

6. Wiederkehr M: Brown tumor complicating end-stage kidney disease . Clin Nephrol Case Stud. 2020, 8:72-9. 10.5414/CNCS110195

7. Salamone D, Muresan S, Muresan M, Neagoe R: Multilevel brown tumors of the spine in a patient with severe secondary hyperparathyroidism A case report and review of the literature. Ann Ital Chir. 2016, 87:S2239253X16024749.

8. Paradowski M, Bladowska J, Halon A, Paradowski B: Recurrent brown tumor of the vertebral column . Neurol India. 2017, 65:908-9. 10.4103/neuroindia.NI_1060_16

9. Alfawareh MD, Halawani MM, Attia WI, Almusrea KN: Brown tumor of the cervical spines: a case report with literature review. Asian Spine J. 2015, 9:110-20. 10.4184/asj.2015.9.1.110

10. Resic H, Masnic F, Kukavica N, Spasovski G: Unusual clinical presentation of brown tumor in hemodialysis patients: two case reports. Int Urol Nephrol. 2011, 43:575-80. 10.1007/s11255-010-9738-3 